\title{
The Predominant Prognostic Significance of NOTCHI Mutation Defined by Emulsion PCR in Chronic Lymphocytic Leukemia
}

\author{
Katarzyna Skórka' \\ Michał Chojnacki (1D) \\ Marta Masternak (D) \\ Agnieszka Karczmarczyk' \\ Edyta Subocz ${ }^{2}$ \\ Ewa Wawrzyniak ${ }^{3}$ \\ Krzysztof Giannopoulos 1,4 \\ 'Department of Experimental \\ Hematooncology, Medical University of \\ Lublin, Lublin, Poland; ${ }^{2}$ Department of \\ Internal Medicine and Hematology, \\ Military Institute of Medicine, Warsaw, \\ Poland; ${ }^{3}$ Department of Hematology, \\ Medical University of Lodz, Lodz, Poland; \\ ${ }^{4}$ Department of Hematology, St. John's \\ Cancer Centre, Lublin, Poland
}

Purpose: NOTCH1 ${ }^{\text {mut }}$ represents a new prognostic marker in chronic lymphocytic leukaemia (CLL). The low sensitivity of the current methods may increase the risk of false-negative results, particularly in patients with low $N O T C H 1^{\text {mut }}$ allelic burden. This study compared two methods of the $N O T C H 1^{\text {mut }}$ assessment including droplet digital PCR (ddPCR) and amplification-refractory mutation system PCR (ARMS-PCR) untreated CLL patients.

Patients and Methods: This study included 319 untreated CLL patients. Two PCR-based methods; ddPCR and ARMS-PCR were performed to assess the mutational status of NOTCH1. The Mann-Whitney, Fisher's exact test, Kruskal-Wallis, Kaplan-Meier, Log rank tests and multivariate Cox proportional hazard regression model were used to analyze collected data.

Results: We proved that ddPCR increased the detectability of the $N O T C H 1^{\text {mut }}$ compared to ARMS-PCR in CLL (18.55\% vs 6\%). We showed a shorter time to first treatment (TTFT) in the $N O T C H 1^{\text {mut }}$ group of patients compared to the $N O T C H 1^{\text {wt }}$ defined by ddPCR (1.5 vs 33 months, $\mathrm{p}=0.01$ ). The TTFT survival curves analysis in subgroups divided according to the mutational status of IGHV and NOTCHI assessed by ddPCR discriminated group with the best prognosis: $I G H V^{\mathrm{mut}} N O T C H 1^{\mathrm{wt}}$. Multivariate analysis revealed that the mutational status of $I G H V$ represented an independent prognostic factor for TTFT, while $N O T C H 1^{\text {mut }}$ determined by ddPCR constituted as a dependent prognostic factor for TTFT.

Conclusion: The selection of the precise method of $N O T C H 1^{\text {mut }}$ detection as ddPCR might significantly improve prognostic stratification of CLL patient. Assessment of IGHV might be relevant to more accurate discrimination of prognostic groups of CLL patients, especially in harboring $N O T C H 1^{\text {mut }}$ irrespective of the quantity of allelic burden.

Keywords: chronic lymphocytic leukemia, CLL, NOTCH1, droplet digital PCR, ddPCR, amplification-refractory mutation system PCR, ARMS-PCR, prognostic marker

\section{Introduction}

The most important genetic features predicting clinical outcome in chronic lymphocytic leukemia (CLL) include somatic mutations of coding the genes of immunoglobulin heavy chain variable $(I G H V)$ and TP53 gene as well as deletions of the short arm of chromosome 17 (17p-). ${ }^{1}$ To explain the molecular basis of the high heterogeneity of CLL, new molecular and genomic markers have been recently identified. $^{2-4}$

So far, the whole genome as well as the exome sequencing identified around 80 recurrently mutated genes in CLL, including genes involved in NOTCH signaling
Correspondence: Krzysztof Giannopoulos Department of Experimental

Hematooncology, Medical University of Lublin, Chodzki I, Lublin, 20-093, Poland

Tel +48814486632

$\mathrm{Fax}+48814486634$

Email krzysztof.giannopoulos@gmail.com 
(NOTCH1, NOTCH2, FBXW7)..$^{5-7}$ The most common mutation occurring in more than $90 \%$ of all CLL patients with a mutation in NOTCH1 (NOTCHI ${ }^{\mathrm{mut}}$ ) is a two basepair frameshift-deletion (c.7541_7542delCT). ${ }^{8}$ This lesion mainly involves the truncation of the C-terminal PEST (peptide sequence that is rich in proline $(\mathrm{P})$, glutamic acid (E), serine (S) and threonine (T)) domain, which is associated with turnover of NOTCH1 protein preserving the stable activated form of NOTCH1.,

The incidence of $N O T C H 1^{\text {mut }}$ in CLL patients is variable and depends mainly on the detection method ranging from $7 \%$ to $22 \%{ }^{8,10-14}$ In Richter transformation and chemorefractory CLL, the frequency of $\mathrm{NOTCHI}^{\text {mut }}$ was significantly higher and accounted for up to $20.8 \%$ and $31 \%$, respectively. ${ }^{7,8,15-17}$ Additionally, a higher incidence of this mutation in CLL patients with a trisomy 12 suggests possible functional synergy. ${ }^{18-20}$ Moreover, NOTCHI ${ }^{\text {mut }}$ represented a risk factor of transformation into diffuse large B-cell lymphoma (DLBCL). ${ }^{21}$ Although $\mathrm{NOTCH}_{1}{ }^{\text {mut }}$ was found in the most reports to be associated with a worse prognosis of the disease, there are still limited data about the clinical significance of NOTCH $^{\text {mut }}$ allelic burden. ${ }^{10,12,22}$

In addition to Sanger and NGS sequencing, also methods based on polymerase chain reaction (PCR), including amplification-refractory mutation system (ARMS-PCR) and droplet digital PCR (ddPCR) could be used to detect mutation in the NOTCH1. The ddPCR provides not only qualitative but also quantitative analysis; thus, it might be used to distinguish groups of patients with different $N O T C H I^{\text {mut }}$ allelic burden. In ddPCR absolute quantities are measured by counting molecules of nucleic acid reaction encapsulated in volumetrically defined droplets. ${ }^{23}$ Applications of ddPCR include detection as well as precise and sensitive quantification of low abundance target including rare mutations and gene expression. ${ }^{24,25}$

The utilization of methods with low sensitivity may increase the incidence of false-negative results especially in patients with low $N O T C H 1^{\text {mut }}$ allelic burden. Assessment of $\mathrm{NOTCH}^{\text {mut }}$ distribution in different biological and clinical subgroups of CLL using ddPCR might determine its correlation with the heterogeneous behavior of the disease. The current study aimed to compare the clinical consequences of the ARMS-PCR and ddPCR methods to assess $N O T C H 1^{\text {mut }}$ in CLL patients.

\section{Patients and Methods \\ Characteristics of Patients}

The current study involved 319 (193 males, 126 females, median age 65) newly diagnosed and previously untreated CLL patients at Department of Hematology, St. John's Cancer Centre, Lublin, Department of Hematology, Military Institute of Medicine, Warsaw, as well as Department of Hematooncology and Bone Marrow Transplantation, Lublin. Among a cohort of CLL 319 patients, 300 were included in ARMS-PCR and 248 in ddPCR assessment of $\mathrm{NOTCH}^{\mathrm{mut}}$. In the cohort of 225 , both methods were performed. The clinical characteristics of the three cohorts of CLL patients are presented in Table 1. Cytogenetic aberrations were performed in diagnostic laboratory according to their routine procedures.

\section{Isolation of Mononuclear Cells and DNA}

Peripheral blood mononuclear cells (PBMCs) were isolated by density gradient centrifugation with Biocoll (Biochrom, Berlin, Germany).

DNA was isolated using the Qiamp DNA Blood Mini Kit (Qiagen, Hilden, Germany) according to the manufacturer's protocol. The quality and quantity of the obtained DNA were quantified spectrophotometrically (OD 260/ 280) using a BioSpec-nano (Shimadzu, Yoko, Japan).

\section{Amplification-Refractory Mutation System PCR (ARMS-PCR)}

For the investigation of $N O T C H I^{\text {mut }}$ by ARMS-PCR the Veriti thermal cycler (Thermo Fisher, Waltman, USA) was used. Three starters were used including: 5'GTGACCGCAGCCCAGTT-3' (forward primer for wildtype); 5'TCCTCACCCCGTCCCGA3' (forward primer for mutation); 5'-AAGGCTTGGGAAAGGAAGC-3' (reverse primer for wild-type and mutation). The reaction was conducted in a total volume of $20 \mu \mathrm{L}$ containing Qiagen Multiplex Master Mix (Qiagen, Hilden, Germany), primers and 200 ng DNA under the following conditions: denaturing step at $95^{\circ} \mathrm{C}$ for 3 min followed by 30 cycles at $95^{\circ} \mathrm{C}$ (30 seconds per cycle), annealing step at $57^{\circ} \mathrm{C}(40$ seconds per cycle), and extension at $72^{\circ} \mathrm{C}$ (40 seconds per cycle). Products of PCR reactions were separated in agarose gel during electrophoresis.

\section{Droplet Digital PCR (ddPCR)}

For the investigation of NOTCHI ${ }^{\text {mut }}$ by ddPCR method, the QX200 micro drop reader (Bio-Rad, California, USA) and 
Table I Clinical Characteristic of Patients

\begin{tabular}{|c|c|c|c|}
\hline & $\begin{array}{c}\text { ARMS-PCR } \\
n=300\end{array}$ & $\begin{array}{c}\text { ddPCR } \\
n=248\end{array}$ & $\begin{array}{c}\text { ARMS-PCR } \\
\begin{array}{c}\text { + ddPCR } \\
n=225\end{array}\end{array}$ \\
\hline \multicolumn{4}{|l|}{ Sex } \\
\hline Male & 180 & 149 & 134 \\
\hline Female & 120 & 99 & 91 \\
\hline \multicolumn{4}{|l|}{ Age } \\
\hline Median & 66 & 66 & 66 \\
\hline Range & $38-90$ & $38-90$ & $38-90$ \\
\hline \multicolumn{4}{|l|}{ Rai Stage } \\
\hline 0 & 75 & 56 & 50 \\
\hline I & 37 & 37 & 30 \\
\hline II & 52 & 39 & 37 \\
\hline III & 11 & 10 & 8 \\
\hline IV & 19 & 9 & 9 \\
\hline $\mathrm{n} / \mathrm{a}$ & 106 & 97 & 91 \\
\hline \multicolumn{4}{|l|}{ ZAP-70 (cut off $20 \%$ ) } \\
\hline+ & 84 & 72 & 67 \\
\hline- & 165 & 124 & 115 \\
\hline \multicolumn{4}{|l|}{ CD38 (cut off $30 \%$ ) } \\
\hline+ & 74 & 60 & 58 \\
\hline- & 182 & 144 & $13 \mid$ \\
\hline \multicolumn{4}{|l|}{$\begin{array}{l}\text { Mutational status of } \\
\text { IGHV }\end{array}$} \\
\hline Mutated & 140 & 107 & 106 \\
\hline Unmutated & 157 & 117 & 116 \\
\hline$n / a$ & 3 & 25 & 3 \\
\hline \multicolumn{4}{|l|}{$\begin{array}{l}\text { Cytogenetic } \\
\text { aberrations }\end{array}$} \\
\hline Adverse & 40 & 27 & 26 \\
\hline Intermediate & 91 & 78 & 76 \\
\hline Favorable & 54 & 46 & 46 \\
\hline $\mathrm{n} / \mathrm{a}$ & 115 & 97 & 77 \\
\hline
\end{tabular}

Notes: Cytogenetic aberration subgroups: adverse (I7p-, I Iq-), intermediate (trisomy 12, normal karyotype, various); favorable (isolated 13q-).

Abbreviation: n/a, not available data.

specific molecular probes were used. The mutation in the NOTCH1 gene was detected using PrimePCR ddPCR Mutation Assay: NOTCH $1^{\mathrm{wt}}$ and PrimePCR ddPCR Mutation Assay: NOTCHI ${ }^{\text {mut }}$ (Bio-Rad, California, USA), a set of specific molecular probes for the wild-type and mutation. The ddPCR reaction was conducted using $200 \mathrm{ng}$ template DNA in a final volume of $20 \mu \mathrm{L}$. PCR reaction was performed under the following conditions: denaturing step at $95^{\circ} \mathrm{C}$ for $10 \mathrm{~min}$ followed by 40 cycles at $95^{\circ} \mathrm{C}$ (30 seconds per cycle), annealing/extension step at $55^{\circ} \mathrm{C}$ (60 seconds per cycle). After amplification, the plate was placed into the
QX200 microdrop reader (Bio-Rad, California, USA). Afterward, the data were analyzed using the QuantaSoft v1.7.4. Software (Figure S1A).

\section{Cloning of NOTCHI ${ }^{\text {mut }}$ Fragment}

Genomic DNA isolated from a $N O T C H 1^{\text {mut }}$ patient was amplified using primers: 5'TCCTCACCCCGTCCCGA3' (Fmut primer) and 5'-AAGGCTTGGGAAAGGAAGC-3' Rmut primer). Products were resolved on 1.5\% agarose gels by electrophoresis and purified by QIAquick Gel Extraction Kit (Qiagen, Hilden, Germany) before performing the cloning reaction. Cloning was conducted with the StrataClone PCR Cloning Kit (Agilent Technologies, Santa Clara, USA) according to the manufacturing protocol. Specific NOTCH1 ARMS-PCR was performed to detect the mutation in positive colonies. Mutation carrying clones were also confirmed by Sanger sequencing. The verified $N O T C H 1^{\text {mut }}$ insert was used as a template for evaluation of the detection limit (Figure S1B-D).

\section{Statistical Analysis}

All the results are presented as median values for continuous variables and frequency for categorical variables. Data were being considered significant if the p-value was 0.05 or less. To compute the correlations of variables, Spearman's rank correlation test was used. To evaluate the differences between the continuous variables in subgroups of patients, the MannWhitney test was used. To evaluate the differences between the categorical valuables in subgroups of patients, the Kruskal-Wallis test was conducted. Survival curves of CLL patients were calculated for the time to first treatment (TTFT) according to the Kaplan-Meier method using a Log rank test. A Cox proportional-hazards regression was used to define prognostic variables. As quantitative information on the significance of results $95 \%$ intervals $(95 \% \mathrm{CI})$ of hazard ratios (HR) were estimated. Statistical analyses were performed using Graph Pad Prism 5 (Graph Pad Software, USA) and MedCalc (MedCalc Statistical Software, Belgium).

\section{Results}

\section{Frequency of the NOTCHI ${ }^{\text {mut }}$ Defined by ddPCR Compared to ARMS-PCR}

ARMS-PCR analysis of NOTCH1 mutation status was performed in a cohort of 300 CLL patients. Using the ARMSPCR methodology we found NOTCH1 $1^{\text {mut }}$ in $18 / 300(6 \%)$ CLL patients. The example of ARMS-PCR analysis for NOTCH $1^{\text {mut }}$ detection is presented in data in Figure S2. 
A

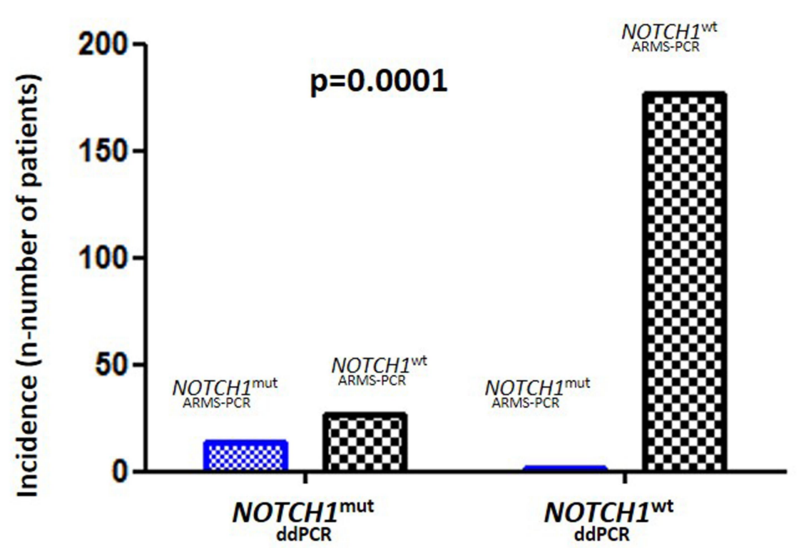

B

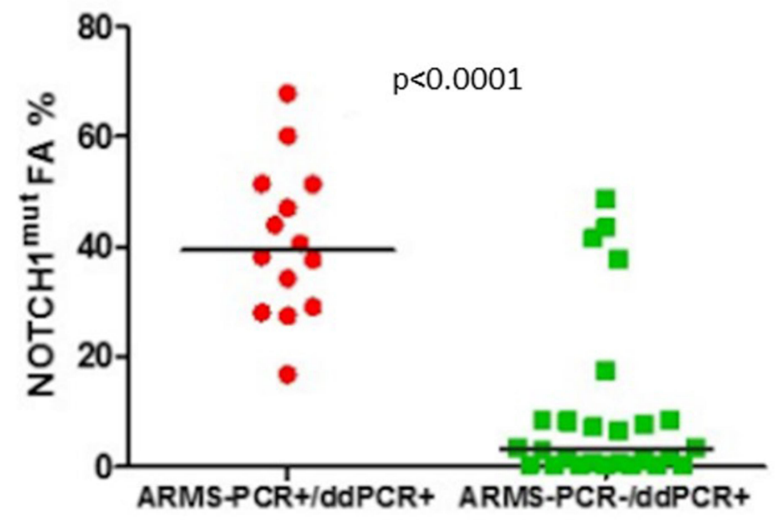

Figure I The coexistence of NOTCHI ${ }^{\text {mut }}$ detection with ddPCR and ARMS-PCR. (A) The coexistence of NOTCHImut detection with ddPCR and ARMS-PCR. Fisher's exact test confirmed the coexistence of NOTCHI mut detection with both methods ( $\mathrm{p}=0.000 \mathrm{I}$ ). The frequency of $\mathrm{CLL}$ patient with $\mathrm{NOTCHI}$ mut assessed by ddPCR was higher than ARMS-PCR (18.55\% vs 6\%). (B) The median NOTCHI mut allelic burden was significantly higher in a group defined as mutated by both ARMS-PCR and ddPCR compared to a group defined as mutated only by ddPCR $(39.45$ vs $3.27 \%, \mathrm{p}<0.000 \mathrm{I})$.

ddPCR analysis of NOTCH1 mutation status was performed in a cohort of 248 CLL patients. The ddPCR analysis determined a higher cohort of CLL patients with NOTCHI ${ }^{\text {mut }} 46 / 248 \quad(18.55 \%)$. The coexistence of NOTCH ${ }^{\text {mut }}$ detection with both methods was confirmed $(\mathrm{p}=0.0001)$ (Figure 1A).

In a cohort of 225 CLL patients, we performed both ddPCR and ARMS-PCR analysis. In this cohort, ARMSPCR detected 16/225 (7.1\%) patients with $N O T C H 1^{\text {mut }}$, while ddPCR revealed 42/225 (18.6\%) with $N O T C H 1^{\text {mut }}$. We found 26/225 (11.6\%) patients with mutation confirmed only by ddPCR, not by ARMS-PCR.

\section{NOTCHI ${ }^{\text {mut }}$ Allelic Burden in CLL}

$N O T C H 1^{\text {mut }}$ allelic burden in $N O T C H 1^{\text {mut }}$ group defined as positive by both ddPCR as well as ARMS-PCR was found to be higher compared to a group of $N O T C H 1^{\text {mut }}$ group assessed as positive only by ddPCR (39.45 vs $3.27 \%$, $\mathrm{p}<0.0001$ ) (Figure 1B). The median of $N O T C H 1^{\text {mut }}$ allelic burden in all CLL patients was $0.016 \%$ with the range of $0-67.9 \%$. The median of $N O T C H 1^{\text {mut }}$ allelic burden in patients with $\mathrm{NOTCH}^{\mathrm{mut}}$ was $7.57 \%$.

\section{Association of NOTCHI ${ }^{\text {mut }}$ Assessed by ddPCR Compared to ARMS-PCR with} Known CLL Prognostic Markers

ARMS-PCR approach showed that $N O T C H 1^{\text {mut }}$ patients are more often characterized by unmutated (UM) IGHV $V^{u m}$ gene status $(\mathrm{n}=17 / 18,95 \%, \mathrm{p}<0.0001)$, compared to NOTCHI ${ }^{\mathrm{wt}}$ patients. Worthwhile ddPCR analysis indicated the only tendency for a more frequent occurrence of $I G H V^{\mathrm{um}}$ in the $N O T C H I^{\mathrm{mut}}$ vs $N O T C H I^{\mathrm{wt}}$ group ( $\mathrm{n}=26 /$ $42,62 \%, \mathrm{p}=0.17$ ) (Figure $2 \mathrm{~A}$ and $\mathrm{B}$ ).

The ddPCR analysis of the NOTCHI ${ }^{\text {mut }}$ assessment showed higher CD38 expression in the $N O T C H 1^{\text {mut }}$ than NOTCH $1^{w t}$ group of CLL patients (24.99 vs 7.48, $<<0.01$ ) (Figure 2C). Moreover, the ddPCR analysis showed a tendency to higher ZAP-70 expression in the $N O T C H 1^{\text {mut }}$ than NOTCH1 ${ }^{\text {wt }}$ group of CLL patients (18.03 vs 10.62, $\mathrm{p}=0.08$ ) (Figure 2D).

Evaluation of $\mathrm{NOTCH}^{\text {mut }}$ using ARMS-PCR showed higher expression of $\mathrm{CD} 38$ (34.43 vs 7.63, $\mathrm{p}=0.02$ ) and ZAP-70 (24.36 vs $11.18, \mathrm{p}=0.03)$ in $N O T C H I^{\text {mut }}$ compared to $\mathrm{NOTCHI}^{\mathrm{wt}}$ group, respectively (Figure $2 \mathrm{E}$ and $\mathrm{F}$ ).

In a cohort of 130 CLL patients, the mutational status of both TP53 and NOTCH1 was assessed. There was no association between status of mutation of NOTCH1 and TP53 ( $\mathrm{p}=0.59)$.

Statistical analysis did not show any associations between NOTCH1 mutational status identified by ddPCR nor by ARMS-PCR and other clinical and biological features, including Rai stage, cytogenetic aberrations and sex.

\section{Prognostic Value of NOTCHI ${ }^{\text {mut }}$ Detected by ddPCR vs ARMS-PCR}

The analysis of survival curves showed a significantly shorter median TTFT in the NOTCHI ${ }^{\text {mut }}$ group of patients 
A

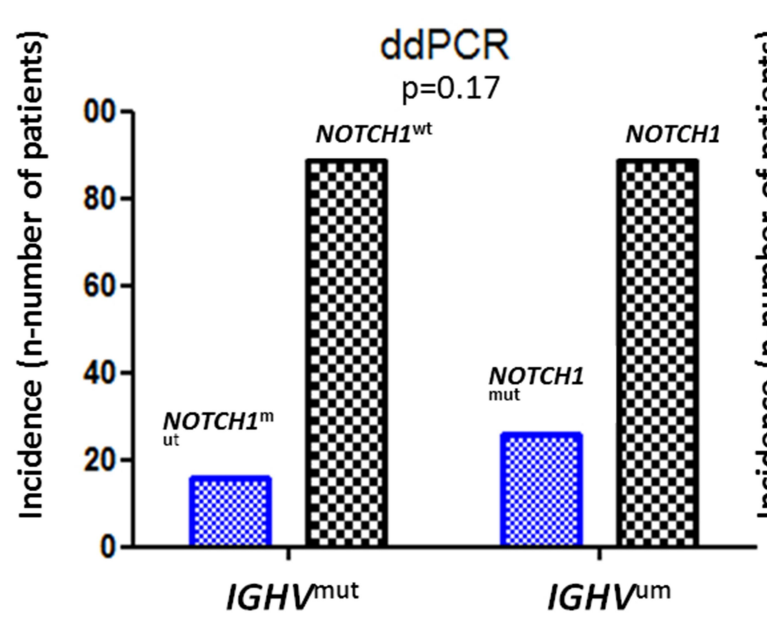

C

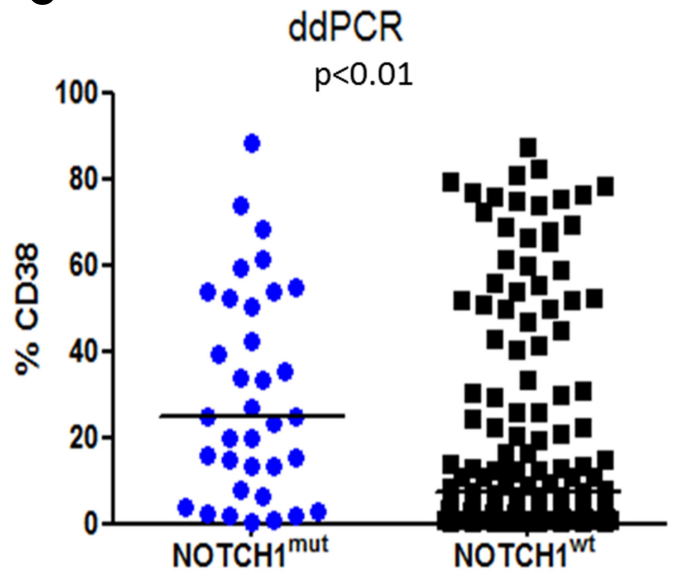

E

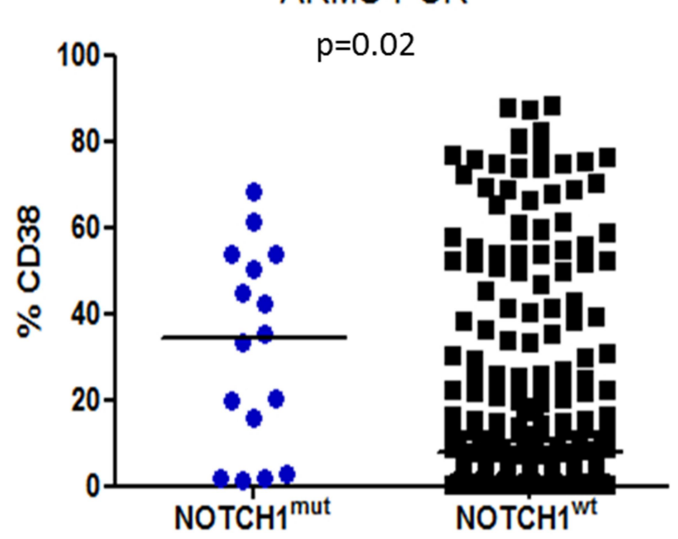

B

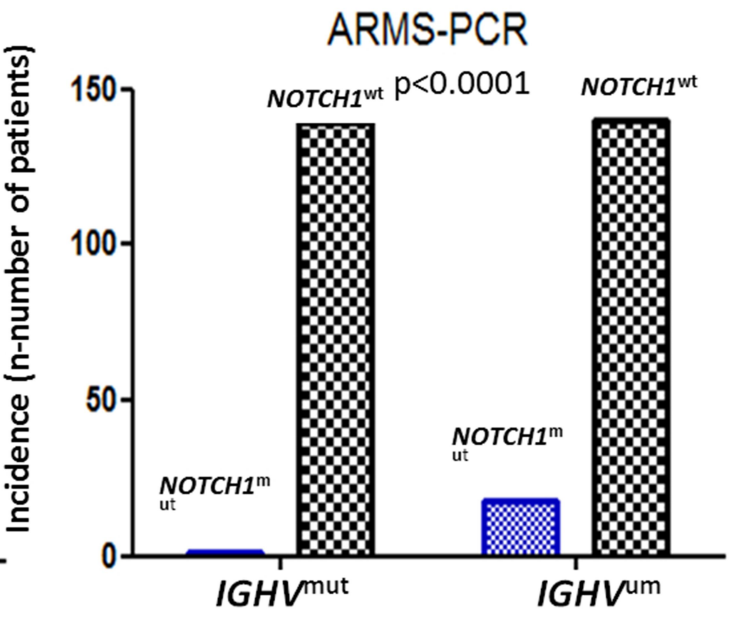

D

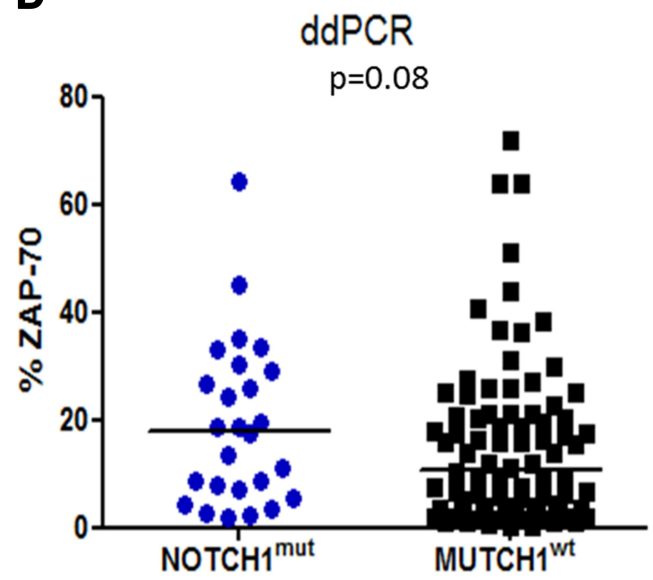

$\mathbf{F}$

ARMS-PCR

$p=0.03$

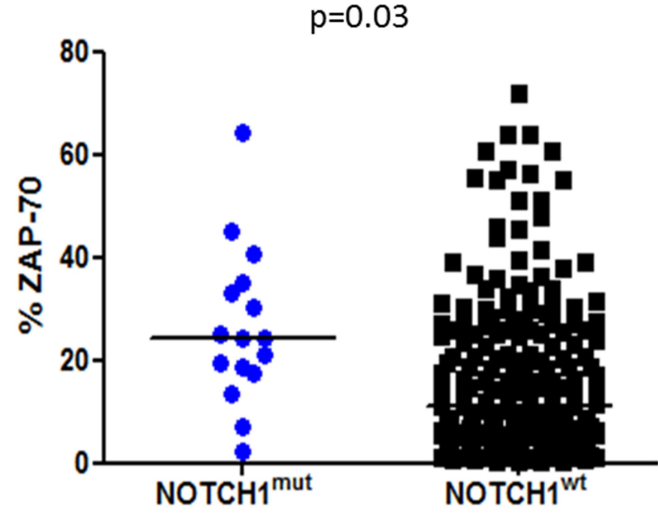

Figure 2 Association of NOTCHI ${ }^{\text {mut }}$ assessed by ddPCR compared to ARMS-PCR with known CLL prognostic markers. (A) The contingency between the mutational status of NOTCHI and IGHV in ddPCR. The ddPCR analysis indicated only a tendency for the more frequent occurrence of unmutated genes (um) for IGHV in the NOTCHI mut vs NOTCH ${ }^{\text {wt }}$ group $(n=26 / 42,62 \%, p=0.17)$. (B) The contingency between the mutational status of NOTCHI and IGHV in ARMS-PCR. The ARMS-PCR analysis showed that NOTCHI ${ }^{\text {mut }}$ patients were more often characterized by IGHV ${ }^{\mathrm{um}}$ gene status compared NOTCHI ${ }^{\text {wt }}$ patients $(\mathrm{n}=17 / \mathrm{I} 8,95 \%, \mathrm{p}<0.000 \mathrm{I})$. (C) The CD38 expression was higher in NOTCHI ${ }^{\text {mut }}$ group compared to NOTCHI ${ }^{\text {wt }}$ defined using ddPCR $(24.99$ vs $7.48, \mathrm{p}<0.0 \mathrm{I})$. (D) The ZAP-70 expression was tended to be higher in NOTCHI ${ }^{\text {mut }}$ group compared to NOTCHI ${ }^{\text {wt }}$ defined by ddPCR (I8.03 vs $10.62, \mathrm{P}=0.08$ ). (E) The CD38 expression was higher in NOTCHI ${ }^{\text {mut }}$ group compared to NOTCHI ${ }^{\text {wt }}$ assessed by ARMSPCR (34.43 vs 7.63, $\mathrm{p}=0.02)$. (F) The ZAP-70 expression was higher in NOTCHI ${ }^{\text {mut }}$ compared to NOTCHI ${ }^{\text {wt }}$ group assessed by ARMS-PCR $(24.36$ vs II.I8, $\mathrm{p}=0.03)$. 
compared to the $N O T C H 1^{w t}$ group assessed by the ddPCR method (1.5 months vs 33 months, $\mathrm{p}=0.01$ ) (Figure 3A). However, there was no significant difference in TTFT in NOTCH1 $1^{\text {mut }}$ vs $N O T C H 1^{\text {wt }}$ group of patients determined by ARMS-PCR ( $\mathrm{p}=0.2$ ) (Figure $3 \mathrm{~B}$ ).

We also found shorter TTFT in NOTCH1 $1^{\text {mut }}$ group of CLL patients defined as mutated by both methods ddPCR and ARMS-PCR compared to NOTCH1 ${ }^{\text {wt }}$ patients with the median 1 month vs 11 months, respectively $(\mathrm{p}=0.001)$ (Figure 3C). In addition, we showed that cohorts of patients with $N O T C H 1^{\text {mut }}$ defined as positive only by ddPCR and patients with $N O T C H 1^{\text {mut }}$ defined by both methods ddPCR and ARMS-PCR were tended to represented groups with worse prognosis and compared to

\section{A}

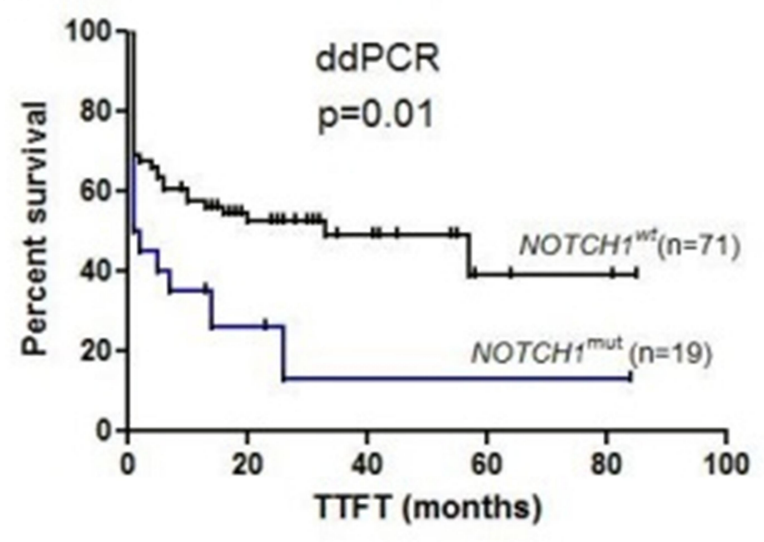

C

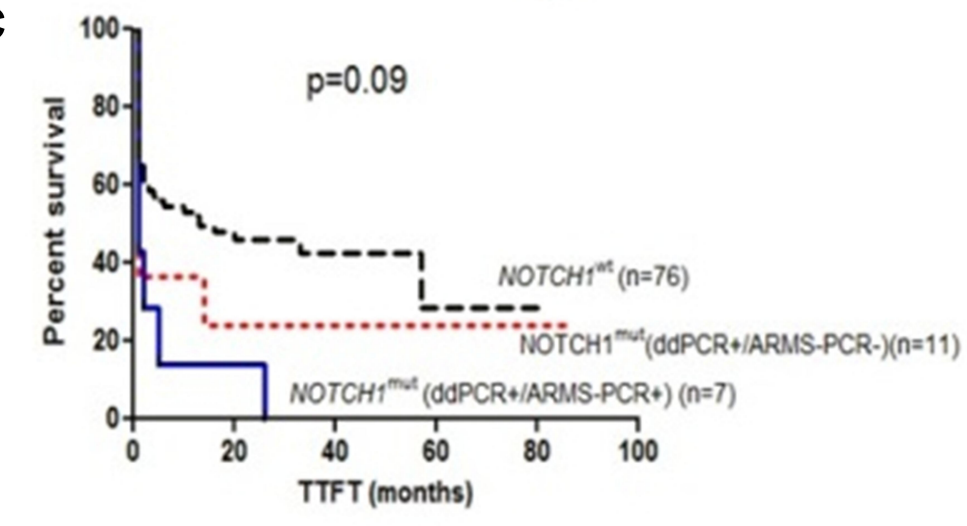

group of $\mathrm{NOTCH}^{\mathrm{wt}} \mathrm{CLL}$ cohort with the median 1 month vs 1 month vs 11 months, respectively $(\mathrm{p}=0.09)$.

\section{Impact of the Mutational Status of NOTCHI Combined with Mutational Status of IGHV on Clinical Outcome in CLL}

In the entire cohort of CLL patients significantly longer TTFT in $I G H V^{m u t}$ cases in which the median TTFT was not reached compared to $I G H V^{u m}$ with the median of 5 months was observed $(\mathrm{p}<0.0001)$ (Figure 4A).

Next, patients were divided into four groups according to the mutational status of IGHV and NOTCHI assessed by

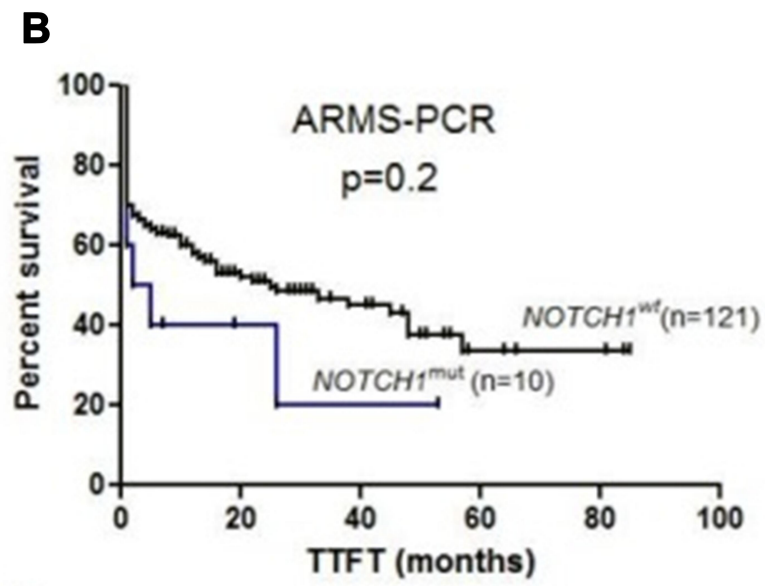

Figure 3 Prognostic value of NOTCHI ${ }^{\text {mut }}$ identified using ddPCR compared to ARMS-PCR. (A) Time to first treatment (TTFT) divided according to the mutational status of NOTCHI assessed by ddPCR. The ddPCR analysis showed significantly shorter TTFT in the NOTCHI mut group of patients compared to NOTCHI ${ }^{\text {wt }}$ (I.5 months vs 33 months, $\mathrm{P}=0.0 \mathrm{I}$ ). (B) TTFT divided according to the mutational status of NOTCHI assessed by ARMS-PCR. No difference was seen in TTFT of NOTCHI mut group of patients compared to NOTCHI ${ }^{\text {wt }}$ determined by ARMS-PCR (3.5 months vs 25 months, $\left.\mathrm{p}=0.2\right)$. (C) TTFT divided into three groups according to the mutational status of NOTCHI identified using ddPCR compared to AMRS-PCR: NOTCHI ${ }^{\text {wt }}$, NOTCHI ${ }^{\text {mut }}$ (ddPCR+/ARMS-PCR+), NOTCHI ${ }^{\text {mut }}$ (ddPCR+/ARMS-PCR-). TTFT is shorter in cases defined as a positive for the detection NOTCHI ${ }^{\text {mut }}$ by both methods: $d d P C R$ and ARMS-PCR compared to those without mutation NOTCHI ${ }^{\text {wt }}$ (I month vs II months, $P=0.00$ I). TTFT in CLL cases bearing NOTCHI ${ }^{\text {mut }}$ defined as mutated only by ddPCR as well as defined as positive by both methods ddPCR and ARMS-PCR was tended to be shorter compared to NOTCH I wt group of CLL (I month vs I month vs II months; $\mathrm{p}=0.09$ ). TTFT between two groups NOTCH Imut detected by ddPCR and not by ARMS-PCR and the NOTCHI ${ }^{\text {wt }}$ group was no statistically different (I month vs II months, $\left.\mathrm{p}=0.25\right)$. TTFT between two groups $N O T C H I^{\text {mut }}$ (ddPCR $+/ A R M S-P C R+$ ) and $N O T C H I$ mut (ddPCR+/ARMS-PCR-) was no statistically different (I month vs I months, $\mathrm{p}=0.32$ ). 
ddPCR: NOTCH1 ${ }^{m u t} I G H V^{m u t}, \quad$ NOTCHI ${ }^{w t} I G H V^{m u t}$, NOTCHI ${ }^{m u t} I G H V^{u m}, \quad N O T C H 1^{w t} I G H V^{u m}$ (Figure 4B). From these four groups, we were able to discriminate a group with the most favorable prognosis: $N O T C H I^{\text {wt }} I G H V^{\text {mut }}$. We proved that among IGHV $V^{\text {mut }}$ cases, TTFT was significantly longer in cases with $\mathrm{NOTCH}^{\mathrm{wt}}$ (median TTFT n.r.) compared to those with harboring NOTCHI ${ }^{\text {mut }}$ (median TTFT $=1$ month). Additionally, we showed that TTFT for $N O T C H 1^{m u t} I G H V^{m u t}$ group was similar to patients with
$I G H V^{u m}$ (median 1 month for NOTCHI ${ }^{m u t} I G H V^{u m}$ group and 2 months for $N O T C H 1^{\mathrm{wt}} I G H V^{\mathrm{um}}$ group).

Almost analogous analyses were performed to characterize the impact of the mutational status of $I G H V$ combined with NOTCH1 determined by ARMS-PCR on clinical outcome in CLL (Figure 4C). Conclusions concerning the prognosis of the one case with $N O T C H I^{\text {mut }} I G H V^{\text {mut }}$ cannot be overinterpreted. Finally, we performed univariate and multivariate analyses for TTFT in reference to the mutational status of $I G H V$ and
A

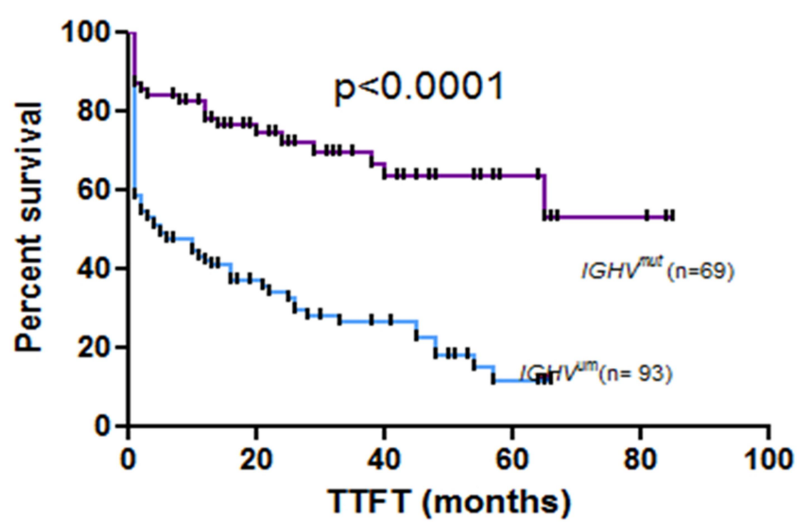

C

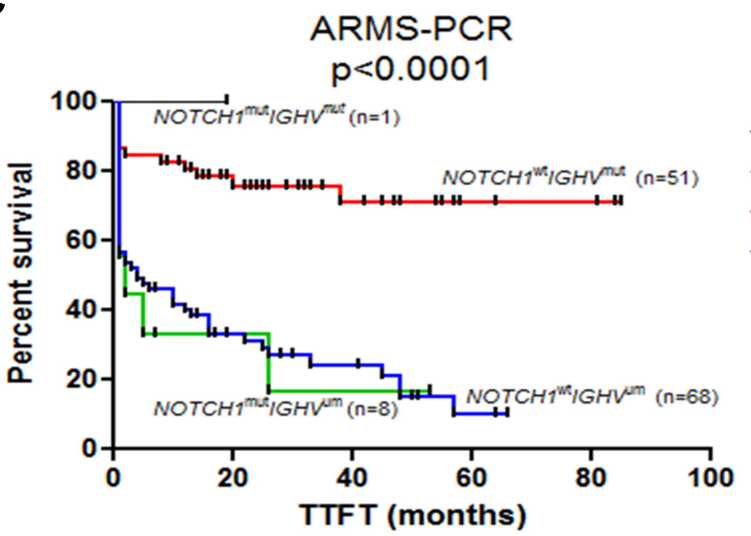

B

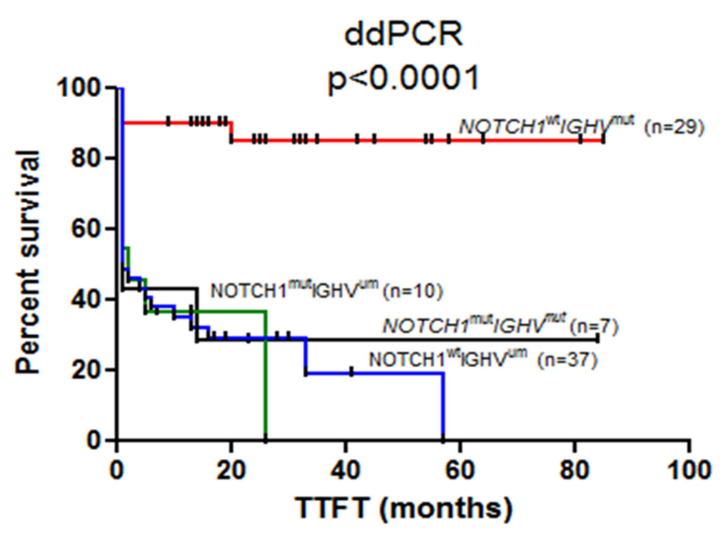

D

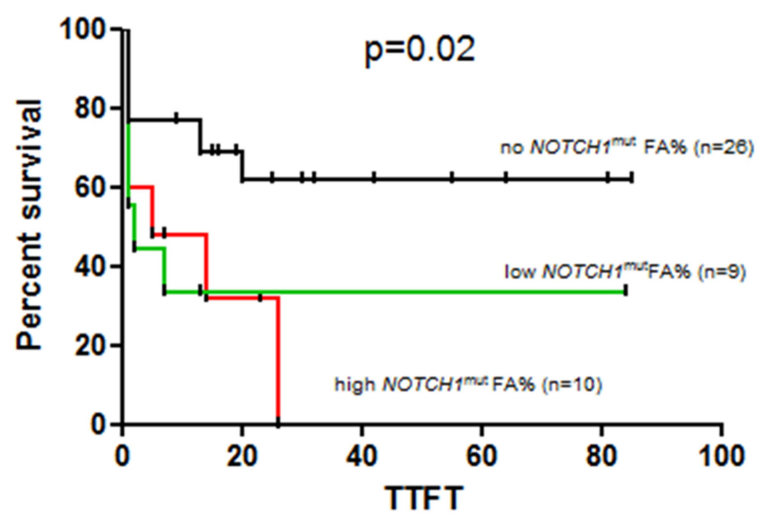

Figure 4 Impact of the mutational status of IGHV and NOTCHI on clinical outcome in CLL. (A) TTFT divided into two groups according to the IGHV mutational status: $I G H V^{\text {mut }} / I G H V^{u m}$. TTFT was longer in IGHV ${ }^{m u t}$ cases in which the median was not reached during the follow-up compared to IGHVm with a median of 5 months ( $<<0.000 \mathrm{I}$ ). (B) TTFT divided into four groups according to the mutational status of IGHV combined with NOTCHI assessed by ddPCR: NOTCHI ${ }^{\text {mut }} / G H V^{\text {mut }}$, NOTCHI ${ }^{\text {wt }} / G H V^{\text {mut }}$, NOTCHI $I^{\text {mut }} / G H V^{u m,}$ NOTCHI $I^{\text {wt } I G H V} V^{u m}$. TTFT was significantly longer in $I G H V^{\text {mut }}$ cases who have also NOTCHI ${ }^{\text {wt }}$ compared to those with harboring NOTCHI mut . The median TTFT was not reached during the follow-up in NOTCHI ${ }^{\text {wt }} / G H V^{\text {mut }}$ cases. The median for NOTCHI ${ }^{\text {mut }} / G H V^{\text {mut }}$ cases was I month. Among the IGHVim cases, the median was I month for NOTCHI mut $/ G H V^{\text {um }}$ group and 2 months for NOTCHI ${ }^{\text {wt }} / G H V^{\text {um }}$ group. (C) TTFT divided into three groups according to the mutational status of IGHV combined with NOTCHI assessed by ARMS-PCR: NOTCHI ${ }^{\text {wt }} / G H V^{\text {mut, }}$ NOTCHI ${ }^{\text {mut }} / G H V^{\mathrm{um},} \mathrm{NOTCH} \mathrm{I}^{\mathrm{wt}} / G \mathrm{GV}^{\mathrm{um}}$. The median was not reached during the follow-up in the NOTCHI $I^{\text {wt }} / G H V^{\text {mut }}$ group. The NOTCHI ${ }^{\text {mut }} / G H V^{\text {mut }}$ group involved only one patient. Among the IGHVm cases, the median TTFT was 2 months for NOTCHImut $/ G H V^{\text {um }}$ group and 4 months for NOTCHI ${ }^{\text {wt }} / G H V^{\text {um }}$ cases. (D) The median TTFT in a group with high NOTCHI ${ }^{\text {mut }}$ allelic burden as well as low NOTCHI ${ }^{\text {mut }}$ allelic burden tended to be shorter compared to the group with no $\mathrm{NOTCH} /{ }^{\text {wt }}$ for whom the median TTFT was not reached during the follow up $(5.5$ months vs 21.5 months, undefined; $\mathrm{p}=0.05)$. The TTFT was shorter in a group with high NOTCHI ${ }^{\text {mut }}$ allelic burden compared to the group with no NOTCH ${ }^{\text {wt }}$ (5 months vs not reached, $\left.\mathrm{p}=0.0 \mathrm{I}\right)$. The TTFT was shorter in a group with low NOTCHI ${ }^{\text {mut }}$ allelic burden compared to the group with no NOTCHI ${ }^{\text {wt }}$ (2 months vs not reached, $\mathrm{p}=0.024$ ). The TTFT was no different between the groups with high NOTCHI ${ }^{\text {mut }}$ allelic burden and low NOTCHI ${ }^{\text {mut }}$ allelic burden ( 5 months vs 2 months, $\mathrm{p}=0.90 \mathrm{I}$ ). The high mutation burden group was defined as having above $7.57 \%$ FA of $\mathrm{NOTCHI} I^{\text {ut }}$. The low mutation burden group was defined as having below $7.57 \%$ FA of NOTCHI ${ }^{\text {mut }}$. No mutation burden group was defined as having below $0 \%$ FA of NOTCHI ${ }^{\text {mut }}$ 
Table 2 Univariate and Multivariate Analyses of TTFT in CLL Patients

\begin{tabular}{|l|c|c|c|c|c|c|c|c|}
\hline \multicolumn{2}{|c|}{ Variable } & \multicolumn{3}{|c|}{ Univariate } & \multicolumn{3}{c|}{ Multivariate } \\
\hline & $\mathbf{N}$ & Events & HR & $\mathbf{9 5 \%}$ Cl & $\mathbf{P}$ & HR & $\mathbf{9 5 \%} \mathbf{~ I ~}$ & P \\
\hline IGHV unmut vs mut & 86 & 46 & 2.55 & $(1.31-4.74)$ & 0.003 & 2.64 & $(1.42-4.91)$ & 0.002 \\
\hline $\begin{array}{l}\text { NOTCHI mut vs unmut } \\
\text { (ddPCR) }\end{array}$ & 86 & 46 & 1.74 & $(0.91-3.32)$ & 0.095 & 1.66 & $(0.88-3.13)$ & 0.117 \\
\hline
\end{tabular}

NOTCH1 assessed by ddPCR (Table 2). Multivariate analysis including $I G H V$ and NOTCHI defined by ddPCR in our cohort of CLL patients revealed that only IGHV unmutated status represented independent factor associated with shorter TTFT (HR, 2.64; 95\%-CI, 1.42-4.91; p=0.002). While the mutational status of NOTCH1 defined by ddPCR constituted a dependent factor on the mutational status of IGHV (HR, 1.66; 95\%-CI, 0.88-3.13; p=0.117).

\section{Prognostic Value of the NOTCHI ${ }^{\text {mut }}$}

\section{Allelic Burden}

To define the prognostic significance of $N O T C H 1^{\text {mut }}$ allelic burden, we analyzed Kaplan-Meier curves for groups with high, low and no NOTCH1 $1^{\text {mut }}$ allelic burden defined as a frequency of fractional abundance (FA\%) obtained in the ddPCR analysis. FA denotes the proportion of the mutant allele frequencies assessed by the program using in ddPCR, ie, QuantaSoft v1.7.4. Software. FA was calculated as a formula: $\mathrm{FA}=$ absolute quantification of mutant clone/absolute quantification of mutant clone + wild type clones. Data were dichotomized according to the median NOTCH1 $1^{\text {mut }}$ allelic burden in $N O T C H 1^{\text {mut }}$ group of CLL patients with the cut off $7.57 \%$ FA. We found that the median TTFT in group with high $N O T C H 1^{\text {mut }}$ allelic burden as well as low NOTCH1 ${ }^{\text {mut }}$ allelic burden was shorter compared to the group with no $\mathrm{NOTCH}^{\text {mut }}\left(\mathrm{NOTCH} 1^{\text {wt }}\right.$ ) allelic burden for whom the median TTFT was not reached during the follow up (5 months vs 2 months, undefined, $\mathrm{p}=0.02$ (Figure 4D).

\section{The Characterization of NOTCHI ${ }^{\text {mut }}$ Allelic Burden in Different Prognostic Groups of CLL}

Owing to the ddPCR method we found a higher NOTCH1 $1^{\text {mut }}$ allelic burden in a CD38+ group compared to CD38- with the median allelic burden 0.0370 vs 0.0120 $(\mathrm{p}=0.02)$. In accordance with increased $N O T C H 1^{\text {mut }}$ allelic burden in ZAP-70+ group compared to ZAP-70- with the median allelic burden 0.0330 vs $0.009(p=0.01)$ was demonstrated.

Analogous analysis in the cohort of only NOTCH1 $1^{\text {mut }}$ samples defined by ddPCR showed a tendency to higher NOTCH $1^{\text {mut }}$ allelic burden in $\mathrm{CD} 38+$ group compared to CD38- (29.1 vs 3.28, $\mathrm{p}=0.10)$. However, there were no statistically significant differences in NOTCH1 mutation allelic burden in a ZAP-70+ group compared to ZAP-70(28.30 vs $5.67, \mathrm{p}=0.381$ ).

Interestingly, NOTCH1 $1^{\text {mut }}$ allelic burden tends to be elevated in male patients compared to female patients with the median allelic burden 0.0210 vs 0.009 ( $\mathrm{p}=0.09)$. We did not observe any differences in $N O T C H 1^{\text {mut }}$ allelic burden in reference to mutation status of $I G H V(\mathrm{p}=0.586)$, TP53 ( $\mathrm{p}=0.11)$, MYD88 ( $\mathrm{p}=0.270)$, SF3B1 ( $\mathrm{p}=0.469)$. We did not find any differences in $N O T C H 1^{\text {mut }}$ allelic burden in patients with $17 \mathrm{p}$ or/and $11 \mathrm{q}$ deletion compared to other patients $(\mathrm{p}=0.966)$. We did not find any association between $\mathrm{NOTCH} 1^{m u t}$ allelic burden with the Rai stage $(p=0.324)$, age $(p=0.520)$, serum level of $\beta_{2}$ microglobulin $(p=0.208)$, and activity of lactate dehydrogenase (LDH) $(\mathrm{p}=0.107)$. The analysis revealed no differences in NOTCH $1^{\text {mut }}$ allelic burden between a group of patients with stereotyped subsets associated with negative prognosis including \#1, \#2, \#8 and other subsets $(\mathrm{p}=0.473)$.

\section{Discussion}

The prognostic role of $\mathrm{NOTCH} 1^{\text {mut }}$ has been investigated during the last few years. ${ }^{10,12,21,26,27}$ There was evidence that patients at progression as well as relapse, had more frequently $N O T C H 1^{\text {mut }} \cdot 8,10,21$ It was confirmed that NOTCH $1^{\text {mut }}$ was predominantly clonal in advanced CLL. Thereby $\mathrm{NOTCH} 1^{\text {mut }}$ has been considered as an early molecular event in the clonal evolution of CLL. ${ }^{28}$

The association of NOTCH1 $1^{\text {mut }}$ with shorter OS and shorter TTFT in CLL proved in many studies ${ }^{10,11,18,22,27}$ while others cannot reach its statistical significance. ${ }^{15,29,30}$ The TTFT is a clinically validated endpoint for treatment-naive CLL 
patients reflecting in a more appreciated way the intrinsic biological complexity of the disease independently on the efficacy of treatment, treatment-induced clonal evolution as well as disease-related and unrelated deaths. Identifying the association between TTFT and some marker might help to identify early drivers in leukemogenesis and progression. ${ }^{31,32}$

Several studies ${ }^{8,10,18,33}$ revealed that $N O T C H 1^{\text {mut }}$ was detected in about $8-10 \%$ of CLL patients at diagnosis using ARMS-PCR. Minervini et $\mathrm{al}^{22}$ also found a higher incidence of $N O T C H 1^{\text {mut }}$ using ddPCR compared to (allele-specific PCR) AS-PCR in a small cohort of CLL patients $(53.4 \%$ vs $37.5 \%)$. This discrepancy with our results might be explained mainly by the different limits of detection of the ddPCR assay as well as may reflect distinct clinical features of the study group of CLL patients. Minervini et $\mathrm{al}^{22}$ defined the limit of detection of the ddPCR assay as FA $>0.03 \%$, while we calculated the limit of detection as FA $>0.06 \%$. Moreover, Hoofd et $\mathrm{al}^{34}$ using ddPCR showed the high frequency of $N O T C H I^{\text {mut }}$ in a group of unselected CLL patients with the highest onset in a group of patients with trisomy 12 .

In survival analyses, we revealed significantly shorter median TTFT in the NOTCHI ${ }^{\text {mut }}$ group of patients compared to the NOTCHI ${ }^{\mathrm{wt}}$ defined by ddPCR. Moreover, in our cohort of patients, ARMS-PCR was not able to provide a significant difference in TTFT in $\mathrm{NOTCHI}^{\text {mut }}$ group of patients vs $\mathrm{NOTCH}^{\mathrm{wt}}$ although the difference was of 3.5 months vs 25 months. No significant difference in TTFT between two groups $N O T C H 1^{\text {mut }}$ detected by ddPCR and not by ARMS-PCR compared to $N O T C H 1^{\mathrm{wt}}$ group ( 1 month vs 11 months, $\mathrm{p}=0.25$ ) might be a consequence of the limitations of the current paper that are associated with the retrospective design of the study, patient selection due to use of available samples as well as the limited number of events. Moreover, in survival analyses, we showed also that TTFT is shorter in cases defined as a positive for the detection $\mathrm{NOTCHI}^{\text {mut }}$ by both methods: ddPCR and ARMS-PCR compared to those without mutation NOTCHI ${ }^{\mathrm{wt}}$ (1 month vs 11 months, $\mathrm{p}=0.001$ ). Hence, our results might suggest that detection of $N O T C H 1^{\text {mut }}$ by ddPCR could allow predicting a group of patients with an unfavorable prognosis at an earlier stage of the disease. Interestingly, differentiation of survival curves revealed that at 24 months all of the patients from the cohort defined as mutated using ddPCR and ARMS-PCR have initiated the treatment. Whereas in the cohort defined as mutated by ddPCR only above $20 \%$ patients remained untreated, what might suggest that the allelic burden of $\mathrm{NOTCH}^{\text {mut }}$ tends to have prognostic value as ARMS-PCR have low sensitivity and detects mutation only in patients with high level of $N O T C H 1^{m u t}$. We observed that in addition to a group of patients with high NOTCHI $^{m u t}$ allelic burden also a group with low NOTCHI $1^{\text {mut }}$ allelic burden have a worse prognosis than a group with $N O T C H I^{\text {wt }}$. Thereby we might suggest the importance of $\mathrm{NOTCH}^{\text {mut }}$ assessment using ddPCR as a quantitative method in CLL patients in predicting the outcome.

Interestingly, Lionetti et $\mathrm{al}^{35}$ using ultra-deep NGS with high sensitivity for the detection of small mutated subclones represented in $1 \%$ of the tumor cell population, revealed the occurrence of $\mathrm{NOTCH}^{\text {mut }}$ in a considerable cohort of patients with monoclonal B cell lymphocytosis (MBL) or early clinical stage of CLL. They proved that NOTCHI $1^{\text {mut }}$ occurred in $11 \%$ of monoclonal B cell lymphocytosis (MBL) as well as in $13.4 \%$ CLL cases with Binet stage A. Moreover, they found that NOTCH1 mutational burden generally tends to be stable over time in NOTCHI ${ }^{\text {mut }}$ group of patients, and this did not appear during disease progression. ${ }^{35}$ Interestingly, Raponi et $\mathrm{al}^{36}$ analyzed the occurrence of NOTCH1 $1^{\text {mut }}$ in 20 ultra-stable CLL (US-CLL) patients characterized as a group of patients with no progression for over 10 years from the time of diagnosis. Using whole-exome sequencing (WES) analyses, they detected no clonal mutations of NOTCH1 in US-CLL. Additionally, Amin et $\mathrm{al}^{37}$ showed that $N O T C H 1^{\text {mut }}$ was already clonal before the therapy indicating the pretreatment driver role in the pathogenesis of CLL. Similarly other studies indicated that the acquisition of mutation during the CLL evolution is uncommon. ${ }^{7,14,21}$ Although in cases progressing to Richter Syndrome or chemorefractory CLL $N O T C H I^{\text {mut }}$ allelic burden was found to not be stable during the disease course. ${ }^{8,10}$ In addition, Minervini et $\mathrm{al}^{22}$ observed that $\mathrm{NOTCHI}^{\text {mut }}$ allelic burden was reduced significantly after therapy.

Taking into consideration rather stable mutational burden of NOTCH1 during the disease course of CLL, we provide the significant role of ddPCR analysis including qualitative and quantitative of NOTCH1 mutational status at the time of diagnosis to discriminate group of patients with low NOTCH1 ${ }^{\text {mut }}$ allelic burden that cannot be detected as a mutant by conventional ARMS-PCR. Further results obtained by Sportoletti et $\mathrm{al}^{18}$ showed that new high-sensitivity AS-PCR represents a valid tool for prognostic screening in CLL with higher sensitivity (0.1\%) than direct Sanger Sequencing (10\%). They observed that 
sequential samples derived from three patients became positive by both methods during the progression of the disease, suggesting that subclonal NOTCH1 mutation was progressively selected to a clonal level. Authors also proved that $\mathrm{NOTCH}{ }^{\mathrm{mut}}$ cases defined as positive only by the AS-PCR have shorter OS than NOTCHI ${ }^{\text {wt }}$.

Despite the great sensitivity, ddPCR detects only the c.7541-7542celCT point mutation, missing all other coding and $3^{\prime} \mathrm{UTR}$ mutations in contrast to time-consuming method of the NGS sequencing. ${ }^{38}$ In the latest NGS analysis, Hu et $\mathrm{al}^{27}$ correlated 29 somatic mutations including NOTCH1, IGHV, TP53 with TTFT in treatment-naive CLL patients and proved at univariable analyses shorter TTFT referring to $N O T C H I^{\mathrm{mut}} I G H V^{\mathrm{um}}$. They suggested that $I G H V^{\mathrm{um}}$, as well as $N O T C H 1^{\mathrm{mut}}$ might be of particular importance in early disease progression in contrast to TP53 $3^{\text {mut }}$.

The International prognostic score (IPS-E) for TTFT prediction of asymptomatic early-stage chronic lymphocytic leukemia includes the analysis of the mutational status of $I G H V$ genes. Patients with mutated $I G H V$ have a favorable prognosis in contrast to patients with unmutated $I G H V$ with unfavorable prognosis. ${ }^{39}$ In our study, survival analysis enables to discriminate a group with the most favorable prognosis: NOTCHI ${ }^{\mathrm{wt}} I G H V^{\mathrm{mut}}$. We proved that TTFT for $N O T C H 1^{\mathrm{wt}} I G H V^{\mathrm{mut}}$ group was significantly longer compared to $N O T C H I^{\text {mut }} I G H V^{\text {mut }}$. Moreover, the prognosis of $N O T C H I^{\text {mut }} I G H V^{\text {mut }}$ group was similar to $I G H V^{u m}$ cases including NOTCHI ${ }^{\mathrm{mut}} I G H V^{\mathrm{um}} N O T C H 1^{\mathrm{wt}} I G H V^{\mathrm{um}}$. Analogical analyses with the use of ARMS-PCR did not show such differences in TTFT and analysis of NOTCH1 mutation did not improve $I G H V$-based stratification. Thereby we indicate that the selection of the precise method of $N O T C H I^{\text {mut }}$ detection as ddPCR and assessment of IGHV might be relevant to more accurate discrimination of prognostic groups of CLL patients, especially those harboring $N O T C H 1^{\text {mut }}$ irrespective of the quantity of allelic burden.

In conclusion, ddPCR provides higher sensitivity in NOTCH1 ${ }^{\text {mut }}$ detection in CLL. Application of ddPCR for patients with CLL at the time of diagnosis might enable earlier identification of a group of patients harboring a low burden of $\mathrm{NOTCHI}^{\mathrm{mut}}$ associated with a worse prognosis. Analysis of the mutational status of $I G H V$ combined with NOTCH1 assessed by ddPCR could discriminate group with the best prognosis: $I G H V^{\mathrm{mut}} \mathrm{NOTCH}{ }^{\mathrm{wt}}$.

Our data suggest the relevance of using a very sensitive method of ddPCR to detect NOTCHI ${ }^{\text {mut }}$ to improve prognostic stratification of CLL patients, especially newly diagnosed and treatment naïve CLL patients. Consequently, the cooperation molecular markers with the clinical features might appoint treatment decisions and thereby applicate precision medicine in the treatment algorithm of CLL. ${ }^{40}$

\section{Data Sharing Statement}

The datasets used and analyzed during the current study are available from the corresponding author on reasonable request.

\section{Ethics Approval and Informed Consent}

The study was conducted according to the guidelines of the Declaration of Helsinki, and approved by the Local Ethics Committee of the Medical University of Lublin (number KE-0254/231/2015), and the informed consent was obtained from all patients and patient information was anonymized and de-identified prior to analysis.

\section{Acknowledgments}

Authors would like to thank persons involved in collecting patients' material and clinical data including: Waldemar Tomczak, Joanna Zaleska, Joanna Purkot, Magdalena Paziewska, Marta Karp, Grażyna Stasiak, Maciej Putowski, Jacek Zawiślak, Joanna Knap, Ewelina Zakrzewska.

\section{Author Contributions}

All authors made a significant contribution to the work reported, whether that is in the conception, study design, execution, acquisition of data, analysis and interpretation, or in all these areas; took part in drafting, revising or critically reviewing the article; gave final approval of the version to be published; have agreed on the journal to which the article has been submitted; and agree to be accountable for all aspects of the work.

\section{Funding}

This research was funded by the Medical University of Lublin, educational grant number MNmb536 and DS462 as well as grant of the Polish Scientific Centre (NCN 2018/ 29/B/NZ5/02706).

\section{Disclosure}

The authors report no conflicts of interest in this work. 


\section{References}

1. Hallek M, Cheson BD, Catovsky D, et al. iwCLL guidelines for diagnosis, indications for treatment, response assessment, and supportive management of CLL. Blood. 2018;131(25):2745-2760. doi:10.1182/blood-2017-09-806398

2. Sharma S, Rai KR. Chronic lymphocytic leukemia (CLL) treatment: so many choices, such great options. Cancer. 2019;125 (9):1432-1440. doi:10.1002/cner.31931

3. Parikh SA. Chronic lymphocytic leukemia treatment algorithm 2018. Blood Cancer J. 2018;8(10):93. doi:10.1038/s41408-018-0131-2

4. Mosquera Orgueira A, Antelo Rodríguez B, Alonso Vence N, et al. Time to treatment prediction in chronic lymphocytic leukemia based on new transcriptional patterns. Front Oncol. 2019;9:79. doi:10.3389/ fonc.2019.00079

5. Puente XS, Pinyol M, Quesada V, et al. Whole-genome sequencing identifies recurrent mutations in chronic lymphocytic leukaemia. Nature. 2011;475(7354):101-105. doi:10.1038/nature10113

6. Quesada V, Conde L, Villamor N, et al. Exome sequencing identifies recurrent mutations of the splicing factor SF3B1 gene in chronic lymphocytic leukemia. Nat Genet. 2012;44(1):47-52. doi:10.1038/ ng. 1032

7. Rossi D, Rasi S, Spina V, et al. Integrated mutational and cytogenetic analysis identifies new prognostic subgroups in chronic lymphocytic leukemia. Blood. 2013;121(8):1403-1412. doi:10.1182/blood-2012$09-458265$

8. Fabbri G, Rasi S, Rossi D, et al. Analysis of the chronic lymphocytic leukemia coding genome: role of NOTCH1 mutational activation. J Exp Med. 2011;208(7):1389-1401. doi:10.1084/jem.20110921

9. Rosati E, Baldoni S, De Falco F, et al. NOTCH1 aberrations in chronic lymphocytic leukemia. Front Oncol. 2018;8:229. doi:10.3389/fonc.2018.00229

10. Rossi D, Rasi S, Fabbri G, et al. Mutations of NOTCH1 are an independent predictor of survival in chronic lymphocytic leukemia. Blood. 2012;119(2):521-529. doi:10.1182/blood-2011-09-379966

11. Baliakas P, Hadzidimitriou A, Sutton L-A, et al. Recurrent mutations refine prognosis in chronic lymphocytic leukemia. Leukemia. 2015;29(2):329-336. doi:10.1038/leu.2014.196

12. Sportoletti P, Baldoni S, Cavalli L, et al. NOTCH1 PEST domain mutation is an adverse prognostic factor in B-CLL. $\mathrm{Br} J$ Haematol. 2010;151(4):404-406. doi:10.1111/j.1365-2141.2010.08368.x

13. Nadeu F, Delgado J, Royo C, et al. Clinical impact of clonal and subclonal TP53, SF3B1, BIRC3, NOTCH1, and ATM mutations in chronic lymphocytic leukemia. Blood. 2016;127(17):2122-2130. doi:10.1182/blood-2015-07-659144

14. Jethwa A, Hüllein J, Stolz T, et al. Targeted resequencing for analysis of clonal composition of recurrent gene mutations in chronic lymphocytic leukaemia. $\mathrm{Br} \quad J$ Haematol. 2013;163(4):496-500. doi:10.1111/bjh.12539

15. Fabbri G, Khiabanian H, Holmes AB, et al. Genetic lesions associated with chronic lymphocytic leukemia transformation to Richter syndrome. J Exp Med. 2013;210(11):2273-2288. doi:10.1084/ jem.20131448

16. Del Poeta G, Dal Bo M, Del Principe MI, et al. Clinical significance of c.7544-7545 delCT NOTCH1 mutation in chronic lymphocytic leukaemia. $B r J$ Haematol. 2013;160(3):415-418. doi:10.1111/ bjh. 12128

17. Rossi D, Rasi S, Spina V, et al. Different impact of NOTCH1 and SF3B1 mutations on the risk of chronic lymphocytic leukemia transformation to Richter syndrome. $B r \quad J$ Haematol. 2012;158 (3):426-429. doi:10.1111/j.1365-2141.2012.09155.x

18. Sportoletti P, Baldoni S, Del Papa B, et al. A revised NOTCH1 mutation frequency still impacts survival while the allele burden predicts early progression in chronic lymphocytic leukemia Leukemia. 2014;28(2):436-439. doi:10.1038/leu.2013.289
19. Fabbri G, Dalla-Favera R. The molecular pathogenesis of chronic lymphocytic leukaemia. Nat Rev Cancer. 2016;16(3):145-162. doi:10.1038/nrc.2016.8

20. Puente XS, Beà S, Valdés-Mas R, et al. Non-coding recurrent mutations in chronic lymphocytic leukaemia. Nature. 2015;526 (7574):519-524. doi:10.1038/nature14666

21. Villamor N, Conde L, Martínez-Trillos A, et al. NOTCH1 mutations identify a genetic subgroup of chronic lymphocytic leukemia patients with high risk of transformation and poor outcome. Leukemia. 2013;27(5):1100-1106. doi:10.1038/leu.2012.357

22. Minervini A, Minervini CF, Anelli L, et al. Droplet digital PCR analysis of NOTCH1 gene mutations in chronic lymphocytic leukemia. Oncotarget. 2016;7(52):86469-86479. doi:10.18632/oncotarget.13246

23. Pinheiro LB, Coleman VA, Hindson CM, et al. Evaluation of a droplet digital polymerase chain reaction format for DNA copy number quantification. Anal Chem. 2012;84(2):1003-1011. doi:10.1021/ac202578x

24. Yuan D, Cui M, Yu S, Wang H, Jing R. Droplet digital PCR for quantification of PML-RAR $\alpha$ in acute promyelocytic leukemia: a comprehensive comparison with real-time PCR. Anal Bioanal Chem. 2019;411(4):895-903. doi:10.1007/s00216-018-1508-6

25. Manoj P. Droplet digital PCR technology promises new applications and research areas. Mitochondrial DNA. 2016;27(1):742-746. doi:10.3109/19401736.2014.913168

26. Putowski M, Podgórniak M, Piróg M, et al. Prognostic impact of NOTCH1, MYD88 and SF3B1 mutations in Polish population of chronic lymphocytic leukemia patients. Pol Arch Intern Med. 2017;127(4):238-244.

27. Hu B, Patel KP, Chen H-C, et al. Association of gene mutations with time-to-first treatment in 384 treatment-naive chronic lymphocytic leukaemia patients. $\mathrm{Br} \quad J$ Haematol. 2019;187(3):307-318. doi:10.1111/bjh.16042

28. Landau DA, Carter SL, Stojanov P, et al. Evolution and impact of subclonal mutations in chronic lymphocytic leukemia. Cell. 2013;152 (4):714-726. doi:10.1016/j.cell.2013.01.019

29. Stilgenbauer S, Schnaiter A, Paschka P, et al. Gene mutations and treatment outcome in chronic lymphocytic leukemia: results from the CLL8 trial. Blood. 2014;123(21):3247-3254. doi:10.1182/blood2014-01-546150

30. Oscier DG, Rose-Zerilli MJJ, Winkelmann N, et al. The clinical significance of NOTCH1 and SF3B1 mutations in the UK LRF CLL4 trial. Blood. 2013;121(3):468-475. doi:10.1182/blood-201205-429282

31. Gentile M, Shanafelt TD, Cutrona G, et al. A progression-risk score to predict treatment-free survival for early stage chronic lymphocytic leukemia patients. Leukemia. 2016;30(6):1440-1443. doi:10.1038/ leu. 2015.333

32. Molica S, Mauro FR, Callea V, et al. The utility of a prognostic index for predicting time to first treatment in early chronic lymphocytic leukemia: the GIMEMA experience. Haematologica. 2010;95 (3):464-469. doi:10.3324/haematol.2009.011767

33. Del Giudice I, Rossi D, Chiaretti S, et al. NOTCH1 mutations in +12 chronic lymphocytic leukemia (CLL) confer an unfavorable prognosis, induce a distinctive transcriptional profiling and refine the intermediate prognosis of +12 CLL. Haematologica. 2012;97 (3):437-441. doi:10.3324/haematol.2011.060129

34. Hoofd C, Huang SJ, Gusscott S, et al. Ultrasensitive detection of NOTCH1 c.7544_7545delCT mutations in chronic lymphocytic leukemia by droplet digital PCR reveals high frequency of subclonal mutations and predicts clinical outcome in cases with trisomy 12. J Mol Diagn. 2020;22(4):571-578. doi:10.1016/j.jmoldx.2020.01.008

35. Lionetti M, Fabris S, Cutrona G, et al. High-throughput sequencing for the identification of NOTCH1 mutations in early stage chronic lymphocytic leukaemia: biological and clinical implications. Br J Haematol. 2014;165(5):629-639. doi:10.1111/ bjh. 12800 
36. Raponi S, Del Giudice I, Marinelli M, et al. Genetic landscape of ultra-stable chronic lymphocytic leukemia patients. Ann Oncol. 2018;29(4):966-972. doi:10.1093/annonc/mdy021

37. Amin NA, Seymour E, Saiya-Cork K, et al. Analysis of subclonal and clonal gene mutations before and after therapy in chronic lymphocytic leukemia. Clin Cancer Res. 2016;22(17):4525-4535. doi:10.1158/1078-0432.CCR-15-3103

38. D'Agaro T, Bittolo T, Bravin V, et al. NOTCH1 mutational status in chronic lymphocytic leukaemia: clinical relevance of subclonal mutations and mutation types. Br J Haematol. 2018;182(4):597-602. doi:10.1111/bjh.14843
39. Condoluci A, Terzi Di Bergamo L, Langerbeins P, et al. International prognostic score for asymptomatic early-stage chronic lymphocytic leukemia. Blood. 2020;135(21):1859-1869. doi:10.1182/ blood.2019003453

40. Moia R, Patriarca A, Schipani M, et al. Precision medicine management of chronic lymphocytic leukemia. Cancers (Basel). 2020;12 (3):642. doi:10.3390/cancers 12030642

\section{Publish your work in this journal}

Cancer Management and Research is an international, peer-reviewed open access journal focusing on cancer research and the optimal use of preventative and integrated treatment interventions to achieve improved outcomes, enhanced survival and quality of life for the cancer patient.
The manuscript management system is completely online and includes a very quick and fair peer-review system, which is all easy to use. Visit http://www.dovepress.com/testimonials.php to read real quotes from published authors. 